\title{
PARES REVISORES
}

Hamut'ay 4(1). Enero-junio 2017

\section{Dr. José Bernardo Peña Arcila}

Prof. Titular, Universidad Politécnica Territorial del estado Aragua. Venezuela. Integrante del Grupo internacional de investigación TEIS, Universidad de Granada,

España. Miembro Categoría B, Programa de Estímulo al

Investigador PEII y PPI 9206, Venezuela.

\section{Dr. Víctor M. Hernández Rivero}

Docente e investigador. Laboratorio de educación y nuevas tecnologías (EDULLAB). Dpto. Didáctica e Investigación

Educativa, Universidad de La Laguna. España.

\section{Dr. Walfredo Gonzales Hernández}

Docente investigador, Universidad de Matanzas, Cuba

\section{Dr. Javier Fombona Cadavieco}

Facultad de Formación del Profesorado y Educación.

Docente investigador. Universidad de Oviedo, Espańa.

\section{Dr. Jorge Norberto Cornejo}

Director de proyectos de investigación y extensión.

Gabinete de Desarrollo de Metodologías de la Enseñanza.

Facultad de Ingeniería, Universidad de Buenos Aires, Argentina

\section{Dr. Rafael Andrés Nieto Göller}

Docente investigador. Centro Universitario Internacional,

México. Universidad Simón Bolívar, México.

\section{M. en CTE. Silvia Leticia Fernández Quiroz}

Coordinadora de Proyectos Especiales. Unidad Politécnica para la Educación Virtual. Instituto Politécnico Nacional, México.

\section{Magister María de Jesús Diaz Quintero}

Docente investigador. Centro de Investigación, Desarrollo e Innovación en las TICs - CIDITIC. Universidad Tecnológica de Panamá.

\section{PhD. Héctor Mazurkiewicz}

Profesor ordinario de la Universidad del Zulia (LUZ), Venezuela. Núcleo COL, Licenciado en Educación Mención Industrial (LUZ), Magister en Informática Educativa (Universidad Rafael Belloso Chacín), Doctor en Procesos de Formación en Espacios Virtuales (Universidad de Salamanca - España) e Investigador del Consejo de

Desarrollo Científico y Humanístico (CONDES).
Dr. Ing. Jayguer Vásquez T. Msc. PhD. Coordinador del Grupo de Investigación en Digital Media. Investigador Centro de Investigación, Desarrollo e Innovación en las TICs - CIDITIC, Universidad Tecnológica de Panamá.

\section{Dr. Néstor Fernández}

Docente investigador. Editor en jefe de la Revista de Educación Abierta y a Distancia en México (READ.MX), Universidad Nacional Autónoma de México.

\section{Dra. Melba Stanziola}

Docente investigadora. Facultad de Psicología. Departamento de Psicología Clínica y de la Salud, Universidad de Panamá.

\section{M.Sc. Magda Julissa Rojas Bahamón} Magíster en Ciencias de la Educación. Especialista en Pedagogía. Ingeniera de Sistemas. Docente investigadora Grupo de investigación Lenguajes, representaciones y Educación, Universidad de la Amazonia. Miembro Comité Editorial Revista Amazonia Investiga Universidad de la Amazonia. Docente Titular IE Antonio Ricaurte. Colombia.

\section{Dra. Mercedes Leticia Sánchez Ambriz}

Coordinadora académica del módulo de Educación a distancia, Instituto Latinoamericano de la Comunicación Educativa (ILCE). Miembro del Consejo Editorial de la Revista de la Academia de Educación abierta y a distancia. México. 